\title{
Infrared spectroscopy of planetary nebulae, including Spitzer
}

\author{
J. Bernard-Salas ${ }^{1}$ \\ ${ }^{1}$ Cornell University, 219 Space Sciences Building, Ithaca, NY-14853, USA \\ email: jbs@isc.astro.cornell.edu
}

\begin{abstract}
The final phase of a star's life is crucial for the understanding of the chemical evolution of our Galaxy. The planetary nebula phase offers an excellent opportunity to study the nucleosynthesis history of low- and intermediate-mass stars, and to study the evolution of dust in evolved objects. For these purposes, infrared spectroscopy has been an ideal method to both determine accurate abundances and characterize the dust features that are seen in their spectra. This contribution briefly summarizes some of the main infrared spectroscopic advances in the field during the last few years, with emphasis on the recent results/projects from the Spitzer Space Telescope on galactic and extra-galactic planetary nebulae.
\end{abstract}

Keywords. planetary nebulae: general - infrared: ISM - ISM: abundances - ISM: line and bands, Galaxies: Magellanic Clouds

\section{Introduction}

Planetary Nebulae (PNe) represent one of the latest stages of stellar evolution of stars between one and eight solar masses (Iben \& Renzini 1980). By the ejection of the outer parts of the stellar envelope, the planetary nebula contributes to the elemental enrichment of the interstellar medium. The nebular gas shows lines of many elements allowing the determination of the elemental abundances in the ionized gas. The abundance of elements such as carbon, nitrogen, and oxygen represents the cumulative record of all nucleosynthetic and mixing processes that may have changed the original composition of the gas since the epoch of stellar formation. On the other hand, elements such as sulfur and argon (elements not supposed to change in the course of evolution of low- and intermediate-mass stars) represent the original abundances of the cloud from which their progenitor stars were formed. Infrared (IR) spectroscopy is ideally suited to accurately determine elemental abundances. The use of IR data has several advantages when deriving abundances using only optical and/or UV data (Rubin et al. 1988): 1) Abundances derived from infrared lines are not very sensitive to the adopted electron temperature $\left(T_{\mathrm{e}}\right)$. Furthermore, by combining the infrared lines with the optical and UV lines one can derive the $T_{\mathrm{e}}$ for a larger number of ions giving important insight in the $T_{\mathrm{e}}$ structure in the nebula. 2) Lines of different stages of ionization are emitted in the IR, reducing the uncertainty in the ionization correction factor (ICF). This is specially true for neon, sulfur, and argon. 3) The infrared lines are less affected by extinction.

Moreover, the IR also holds the spectroscopic fingerprints of the dust components present in the outflow, thus allowing to also determine the composition of the dust and certain aspects of dust formation. 


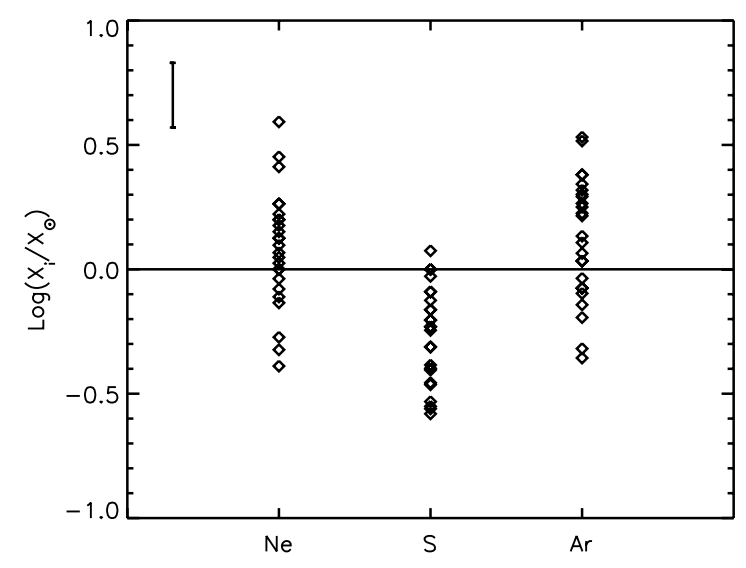

Figure 1. Neon, sulfur and argon abundances of PNe as derived from ISO results. References: Pottasch (1999 through 2005), Kwitter et al. (2003), Bernard-Salas et al. (2001 through 2003), van Hoof et al. (2000), Surendiranath et al. (2004). On the top right a typical error bar is shown.

\section{Recent results before Spitzer}

It is impossible to summarize all the results in IR spectroscopy during the last years in just a few pages. Instead, we highlight some results on PNe using IR spectroscopy with emphasis on the questions that have arisen as a consequence of these studies and that may be resolved with the current/future IR observatories.

\subsection{Abundances}

As mentioned in the introduction, IR spectroscopy is ideal to determine accurate abundances of PNe. Fig. 1 plots the abundances of neon, sulfur and argon for a sample of 25 PNe observed with the Infrared Space Observatory (ISO) with respect to the solar values (Asplund et al. 2005). The abundances were derived mainly from the infrared lines measured by the SWS and LWS spectrometers onboard ISO but were also complemented with optical or UV data in order to have information on all the important stages of ionization. To avoid cramping the bibliography with too many references we have listed these references only in the caption of the figure and one can find the complete references in Pottasch \& Bernard-Salas (2006) (their Table 2). We can see that the neon abundance is roughly solar, although some objects seem to have experienced some enrichment (Marigo et al. 2003). The sulfur abundance is sub-solar. Sulfur and argon are not supposed to be produced or destroyed in the course of evolution of low- and intermediate-mass stars. Indeed the argon abundance in Fig. 1 is close to solar. This sulfur underabundance has been noticed by Kwitter et al. (2003) in a larger sample of PNe using optical data. Henry et al. (2004) discussed in detail the possibilities for such underabundance in their sample and they conclude that most likely explanation resides in the ionization correction factor (ICF) used to account for the (in the optical) unseen S IV. While this may be part of the problem it does not solve it completely because the sulfur abundances shown in Fig. 1 were all derived with ISO using the [S IV] line $(10.5 \mu \mathrm{m})$ and are still sub-solar. Marigo et al. (2003) argue that the solar sulfur abundance may be wrong. This is consistent with the finding of Martín-Hernández et al. (2002) in a large sample of H II regions. They find that the sulfur abundance in their sample is systematically lower than the solar value. Another possibility is that sulfur is depleted into dust. While Savage \& Sembach (1996) 
demonstrate that sulfur is not refractory it is also true that sulfur-based dust is seen in some PNe (e.g. MgS, FeS). Not all PNe with sulfur underabundance show these features but it may be worthwhile to quantify how much sulfur is actually locked up in the dust.

\subsection{Dust}

The spectra of carbon-rich $\mathrm{PNe}(\mathrm{C} / \mathrm{O}>1)$ are dominated by strong emission features due to Polycyclic Aromatic Hydrocarbons (PAHs). Peeters et al. (2002) classified the 6-9 $\mu \mathrm{m}$ $\mathrm{PAH}$ features according to their profile and peak position for a variety of sources. In this classification PNe are mostly class $\mathrm{B} \dagger$ (although there are some PNe with A profiles). PNe show the most variation (peak position) from source to source and this variation is still not understood. Peeters et al. (2002) and Hudgins et al. (2005) suggested that the variation seen in the $6.2 \mu \mathrm{m}$ feature may be the effect of PAH nitrogenation. The effect of PAH nitrogenation is to shift the feature toward the blue but this has not yet been proven. Another interesting feature is the $\mathrm{MgS}$ around $30 \mu \mathrm{m}$. A nice historical review is given by Hony et al. (2002) and summarized here. This feature was discovered by Forrest et al. (1981) in two PNe and several carbon stars. Initially, Goebel \& Moseley (1985) suggested MgS as the carrier. Szczerba et al. (1999) found that the abundances of magnesium and sulfur were consistent with $\mathrm{MgS}$ being the carrier. Hrivnak et al. (2000) and Volk et al. (2002) demonstrated that the feature is actually composed of two subfeatures. While in the meantime other components were proposed as carriers, Hony et al. (2002) successfully modeled the feature in a large sample of evolved objects using MgS. Hony et al. (2002) reproduce the feature in C-rich stars and AGBs, however their model cannot reproduce the narrower profile seen in PNe and this still needs to be explained. This example shows that the identification of dust features in the spectrum is not straightforward and requires major efforts both observationally and with laboratory measurements.

Oxygen-rich PNe usually show silicates in their spectra. Probably, one of the most important discoveries from ISO is the presence of crystalline silicates outside the solar system (for an extensive review see Molster \& Kemper 2005). These were seen in evolved stars (including PNe) by Waters et al. (1996) but also in young stars and comets. The exact composition of these silicates was found to be compatible with pure Mg-olivines (i.e. no iron) (Molster et al. 2002). Also important is the fact that the crystalline silicates seen in PNe and young stars are not the same and they may have a different formation history.

It is important to mention that some PNe show chemistry typical of C-rich and O-rich objects (PAHS, and silicates respectively) such as BD+303639 and NGC 6302. The origin of this dual chemistry is still in debate. Bernard-Salas \& Tielens (2005) suggested that there may be a relationship between the characteristics of the dust emission features and the nucleosynthesis history of the progenitor stars with the PNe with dual chemistry being those experiencing hot-bottom-burning. However this relation can not be proven/rejected because of the uncertainty in the distance to PNe which affects the core-mass determination.

\subsection{Dust and abundances}

A very interesting topic is the relation between the abundances in the gas phase and the dust. Despite its importance little has been done so far in this relation. Back in the 80's Cohen et al. (1986) using the Kuiper Airbone Observatory showed that there is a

$\dagger$ These are sources where the $6.2 \mu \mathrm{m}$ PAH peaks between $6.24-6.28 \mu \mathrm{m}$, the $7.7 \mu \mathrm{m}$ feature is dominated by the $7.8 \mu \mathrm{m}$ complex and the $8.6 \mu \mathrm{m}$ PAH is shifted toward the red (see Peeters et al. 2002 for details). 


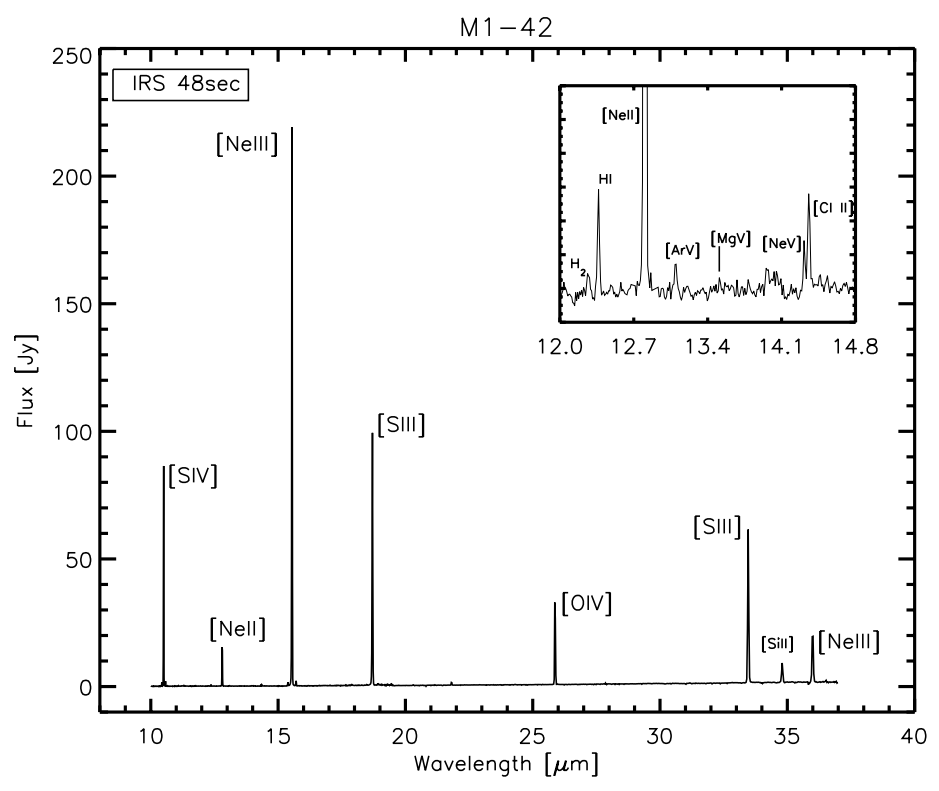

Figure 2. SH and LH spectrum of M1-42. The inset shows a zoom into the $12-15 \mu \mathrm{m}$ region.

correlation between the $7.7 \mu \mathrm{m}$ feature and the $\mathrm{C} / \mathrm{O}$ ratio in the $8 \mathrm{PNe}$ that they studied. This led them to conclude that hydrocarbons are the carriers of these features. Recently, Cohen \& Barlow (2005) have revisited this relation with a larger sample of PNe using ISO data. While there is some scatter they find that the $7.7 \mu \mathrm{m}$ PAH strength to the total IR luminosity ratio correlates with the $\mathrm{C} / \mathrm{O}$ up to a ratio of 3 . For the $11.3 \mu \mathrm{m}$ feature they find a similar relation up to $\mathrm{C} / \mathrm{O}$ ratios of 2 .

\section{Recent results from the Spitzer Space Telescope}

The Spitzer Space Telescope was launched on 2003 August 25 and it has an expected life span of 5.5 years. On board, the InfraRed Spectrograph (IRS) is working extremely well returning outstanding quality data. It consists of four modules: two low-resolution (SL and LL) and two high-resolution (SH and LH). Together they cover the wavelength range from 5 to $38 \mu \mathrm{m}$. Details on the instrument and telescope can be found in Houck et al. (2004) and Werner et al. (2004) respectively. The main feature of the SST is its sensitivity. This is illustrated in Fig. 2 which shows the high resolution spectrum of the bulge PN M 1-42 (Bernard-Salas \& Roellig, private communication) obtained with only 48 seconds on-source time. In the next sections we present the projects devoted to study PNe using IRS (up to date). Most of these projects are still in development and some groups have not yet obtained the data, thus the reader should bear in mind that the majority of the results are preliminary.

\subsection{Bulge PNe}

Bulge PNe have been widely use as a tool to trace the dynamics of the Milky Way (Beaulieu et al. 1979). Their study is of strong relevance in the study of the stellar population of the inner part of the Galaxy. M. Bobrowski has a program to obtain IRS spectroscopy of 61 of these objects. From that sample, Perea Calderón has analyzed those with Wolf-Rayet ([WC-type]) central stars. They find that all the objects, including 
early- and late-type, show carbon-rich (PAHs) and oxygen-rich (crystalline silicate) features in their spectrum (see Perea Calderón proceeding elsewhere in this volume for details). Before, this dual chemistry had only been seen in late-type [WC]PNe. Perea Calderón's finding indicates that the mechanisms responsible for the dual chemistry in $[\mathrm{WC}] \mathrm{PNe}$ are present in both early- and late-type.

\subsection{Halo PNe}

G. Stasinska has a Spitzer program to do IRS spectroscopy on the most metal poor PN, PN G135.9+55.9. K. Kwitter has another program to observe DdDm1, M2-29, and PRMG-1. Of the 10 PNe that have been identified so far to belong to the Galactic Halo (Otsuka et al. 2003), all are metal-deficient (Howard et al. 1997). It has been suggested that their low oxygen abundance may reflect inhomogeneities in the early enrichment of the halo gas (Garnett \& Lacy 1993). The halo PNe offer us the possibility to probe the halo composition of the Milky Way at the epoch of their formation (Torres-Peimbert \& Peimbert 1979; Otsuka et al. 2003). However, it was first observed by Barker (1980) and then by Garnett \& Lacy (1993) that the abundances of sulfur and argon were better probes of the initial metallicity of the stellar progenitor than the oxygen abundance. Realizing this potential, several authors attempted the study of some infrared lines using ground-based observatories. Dinerstein et al. (2003) obtained mid-IR observations with TEXES to detect [SIV] $(10.5 \mu \mathrm{m})$ and [NeII] $(12.8 \mu \mathrm{m})$ in DdDm 1 and H4-1. They detected these lines in DdDm 1 but not in H4-1. Garnett \& Lacy (1993) targeted the [S IV] line at $10.51 \mu \mathrm{m}$ using the NASA IRTF in K 648 and BB-1, but could not detect the line in either object. Spitzer has the sensitivity to obtain full mid-IR spectra of these sources. Kwitter and Stasinska's observations (not yet taken) will certainly improve our understanding of the chemical composition of the Galactic halo.

\section{3. $L M C$ and $S M C$ objects}

One of the main advantages of the SST is that its sensitivity allows the study of PNe outside the Milky Way. Observing targets in the LMC and SMC is particularly interesting because they have known distances and have evolved in lower metallicity environments. The enrichment of the expelled nebular material can be combined with accurate knowledge of the central star's luminosity. Up to the present time, there are two programs to study PNe in the Magellanic Clouds (MC) using IRS. The group of L. Stanghellini obtained IRS spectra of 42 sources in the MC, which they have previously characterized using HST data in terms of morphology, evolution, CS and progenitor masses (see Stanghellini et al. 2002; Villaver et al. 2003, and references therin). They find a relation between the gas phase and dust abundances (see Stanghellini proceeding elsewhere in this volume). J.R. Houck observed 20 PNe, 17 in the LMC and 7 in the SMC. Fig. 4 shows the low-resolution spectra of a subsample by Bernard-Salas et al. (2005, 2006 in prep). The spectra show a large variety of features, ranging from atomic emission-lines to PAHs and amorphous silicates. Some of the objects also show SiC and MgS in their spectrum (SMP LMC 31, 38, 58 and SMP SMC 6). The abundances derived from the fine-structure lines in the limited sample analyzed indicate that the average neon and argon abundances $\left([\mathrm{Ne} / \mathrm{H}]=6.7 \times 10^{-5},[\mathrm{Ar} / \mathrm{H}]=1.1 \times 10^{-6}\right)$ are consistent with the average abundances found in the LMC HII regions $\left([\mathrm{Ne} / \mathrm{H}]=6 \times 10^{-5},[\mathrm{Ar} / \mathrm{H}]=1.4 \times 10^{-6}\right)$ studied by Vermeij \& van der Hulst (2002). The sulfur abundance seems to be underabundant respect to the HII regions. The profiles of the $6.2,7.7,8.6$, and $11.3 \mu \mathrm{m}$ PAH bands show similar variations in peak position as their Galactic counterparts (class B). We don't see evidence for the $6.2 \mu \mathrm{m}$ PAH to peak at shorter wavelengths as one would have expected from a low metallicity environment if its variation was due to PAH nitrogenation. 


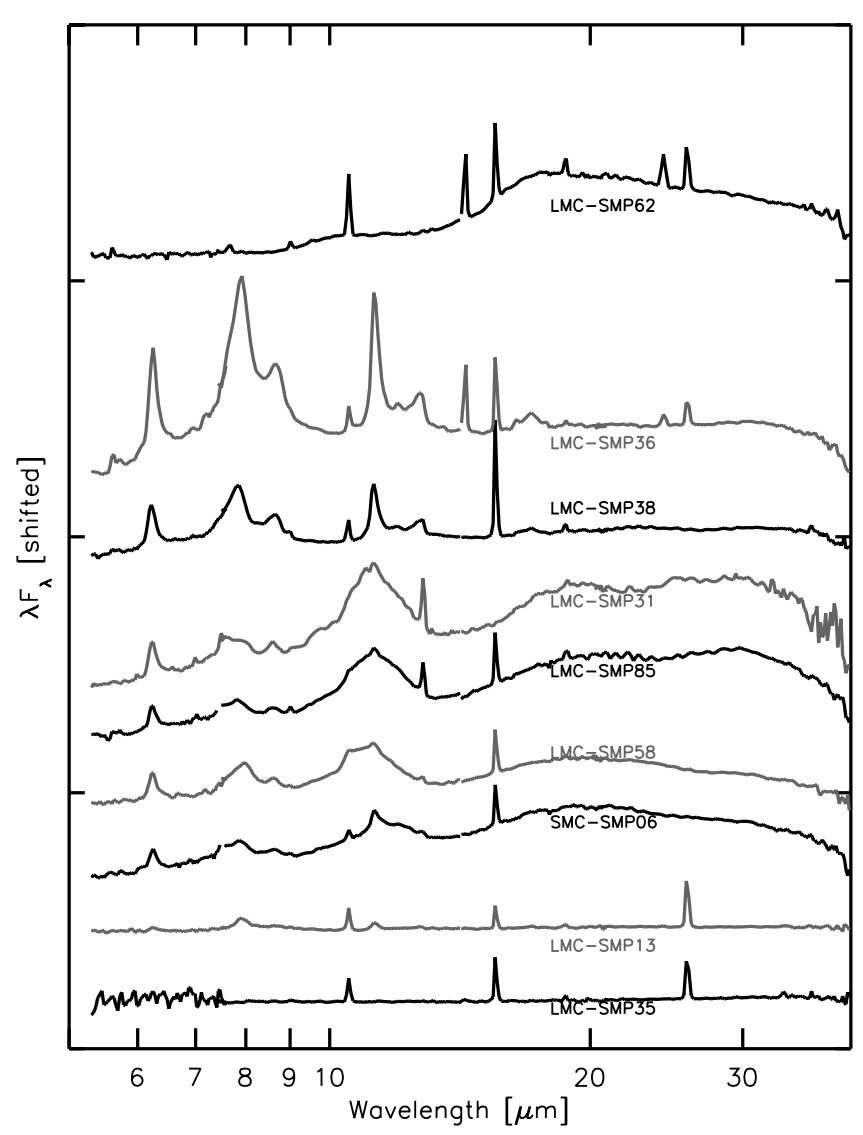

Figure 3. Low resolution spectrum of a sample of MC PNe.

\subsection{Pre-planetary nebulae}

The brief transition between the asymptotic giant phase (AGB) and the PN phase, the pre-planetary nebula $(\mathrm{PPN})$ phase, is critical to understanding the evolution of intermediate-mass stars. R. Sahai has a promising program to study the mass-loss in a sample of 16 PPNe using the IRAC, MIPS and the IRS instruments onboard the SST. In order to model the mass-loss of these objects one needs an accurate representation of the Spectral Energy Distribution (SED). Since most of the nebular mass is cold it emits most of its energy in the infrared and therefore it is ideally suited to be studied with Spitzer.

The PPN phase is also very important to study the dust chemistry. The ISO-SWS spectrum of the PPN AFGL 618 (Cernicharo et al. 2001a, 2001b) showed a very rich chemistry of acetylene, polyacetylenic chains of $\mathrm{C}_{4} \mathrm{H}_{2}$ and $\mathrm{C}_{6} \mathrm{H}_{2}$ benzene, $\mathrm{HCN}$ and $\mathrm{C}_{2} \mathrm{H}_{4}$ to cite some. Cernicharo et al. (2001a) suggest that these molecules are the building blocks from which PAHs are formed. The SST has observed two very interesting objects which show similar features. The IRS spectrum of SMP LMC 11 (Bernard-Salas et al. 2006) is also dominated by molecular absorption (see Fig. 4). This object was previously classified as a PN but clearly the IRS spectrum (which also shows the low excitation line of $[\mathrm{NeII}])$ identifies it in the PPN phase. In addition, the IRS low-resolution spectrum of MSX SMC 29 by Kraemer et al. (2006) shows a band of acetylene together with 


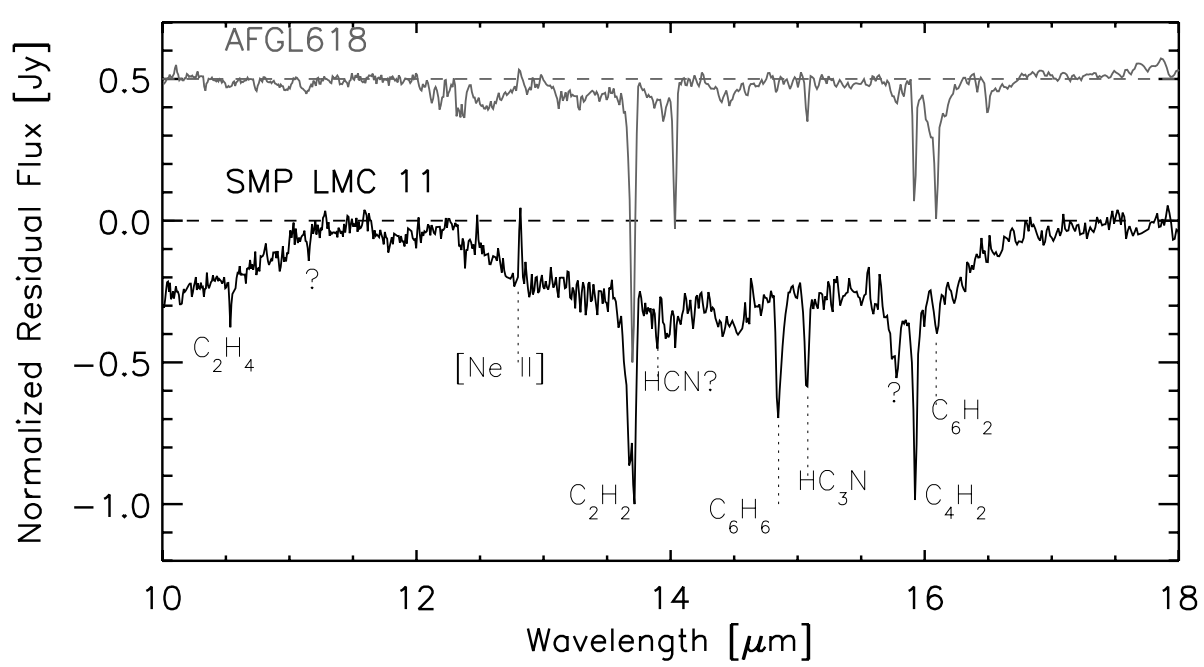

Figure 4. IRS-SH spectrum of SMP LMC 11 (Bernard-Salas et al. 2006) after continuum subtraction. The ISO-SWS spectrum of AFGL 618 is shown in grey for comparison (and shifted 0.5Jy). Both spectra have been normalized for comparison.

$\mathrm{PAH}$ features, that is, the spectrum shows a mixture of PAHs and the PAH progenitor together. SMP LMC 11 and MSX SMC 29, together with AFGL 618, are key objects in the understanding of the chemical paths that lead to PAH formation.

\section{Future airbone/space IR observatories}

The Spitzer Space Telescope will likely be operational until mid 2009. As it has already been shown, its superb sensitivity enables it to study PNe in the MC, and it is capable of obtaining spectroscopy of PNe in the closer members of the Local Group. Following Spitzer, there are 3 airborne/space observatories which will enable IR/sub-mm spectroscopy. SOFIA, the airborne observatory will have nine instruments covering the 0.35 to $655 \mu \mathrm{m}$ region. It high spatial resolution will enable the study of abundance gradients within a nebula. Herschel is expected to be launched in 2008, and the HIFI spectrometer will cover the far-IR and sub-mm region $(60-670 \mu \mathrm{m})$. This instrument will be ideal for studying molecular emission from the PDRs. JWST will be launched no earlier than 2013 and will provide $0.6-27 \mu \mathrm{m}$ coverage with NIRSpec and MIRI. Its large mirror will extend the infrared observation of $\mathrm{PNe}$ to more distant galaxies.

\section{Acknowledgements}

I would like to thank Jan Cami, Stuart Pottasch and Jim Houck for their careful readings of the manuscript.

\section{References}

Asplund, M., Grevesse, N., \& Sauval, A.J. 2005, ASPC 336, 25

Barker, T. 1980, ApJ 240, 99

Beaulieu, S.F., Dopita, M.A., Freeman, K.C., \& Kalnajs A.J. 1997, IAU Symp. 180: Planetary Nebulae pg 405 
Bernard-Salas, J., Peeters, E., Sloan, G.C., Cami, J., \& Houck, J.R. 2006, ApJL in press (astroph/0609299)

Bernard-Salas, J., \& Tielens, A.G.G.M. 2005, A\&A, 431, 523

Bernard-Salas, J., Houck, J.R., Pottasch, S.R., \& Peeters, E. 2005 AIP conference proceedings 804, pg56

Cernicharo, J., Heras, A.M., Tielens, A.G.G.M., et al. 2001a, ApJ 546, L123

Cernicharo, J., Heras, A.M., Pardo, J.R., et al. 2001b, ApJ 546, L127

Cohen, M., \& Barlow, M.J. 2005, MNRAS 362, 1199

Cohen, M., Allamandola, L., Tielens, A.G.G.M., et al. 1986, ApJ 302, 737

Dinerstein, H.L., Richter, M.J., Lacy, J.H., \& Sellgren, K. 2003, AJ 125, 265

Forrest, W.J., Houck, J.R., \& McCarthy, J.F. 1981, ApJ 248, 195

Garnett, D.R., \& Lacy, J.H. 1993, ApJ 419, L93

Goebel, J.H., \& Moseley, S.H. 1985, ApJ 290, L35

Henry, R.B.C., Kwitter, K.B., \& Balick, B. 2004, AJ 127, 2284

Hony, S., Waters, L.B.F.M., \& Tielens, A.G.G.M. 2002, A\&SA 390, 533

Houck, J. R., Appleton, P. N., Armus, L., et al. 2004 ApJS 154, 18

Howard, J.H., Henry, R.B.C., \& McCartney, S. 1997, MNRAS 284, 465

Hrivnak, B.J., Volk, K., \& Kwok, S. 2000, ApJ 535, 275

Hudgins D.M., Bauschlicher, C.W.Jr, \& Allamandola, L.J 2005, ApJ 632, 316

Iben, I., \& Renzini, A. 1983, ARA\&A 21, 271

Kraemer, K.E., Sloan, G.C., Bernard-Salas, J., et al. 2006, ApJL submitted

Kwitter, K.B., Henry, R.B.C., \& Milingo, J.B. 2003, PASP 115, 80

Marigo, P., Bernard-Salas, J., Pottasch, S.R., Tielens, A.G.G.M., \& Wesselius, P.R. 2003, A\&A 409, 619

Martín-Hernández, N.L., Peeters, E., Morisset, C., et al. 2002, A $\& A$ 381, 606

Molster, F., \& Kemper, C. 2005, SSRv ISO special issue 119, 3

Molster, F.J., Waters, L.B.F.M., Tielens, A.G.G.M., Koike, C., \& Chihara, H. 2002, A 8 A 382, 241

Otsuka, M., Tamura, S., Yadoumaru, Y., \& Tajitsu, A. 2003, PASP 115, 67

Peeters, E., Van Kerckhoven, C., Tielens, A.G.G.M., et al. 2002, A\&A 390, 1089

Pottasch, S.R., \& Bernard-Salas, J. 2006 A $\& A$ submitted

Rubin, R.H., Simpson, J.P., Erickson, E.F., \& Haas, M.R. 1988, ApJ 327, 377

Savage, B.D, \& Sembach, K.R. 1996, ARA\&A 34, 279

Stanghellini, L., Shaw, R.A., Mutchler, M., et al. 2002, ApJ 575, 178

Szczerba, R., Henning, T., Volk, K., et al. 1999, A\&A 345, L39

Torres-Peimbert, S., \& Peimbert, M. 1979, RMxAA 4, 341

Volk, K., Kwok, S., Hrivnak, B.J., et al. 2002, ApJ 567, 412

Vermeij, R., van der \& Hulst, J.M. 2002, A\&A 391, 1081

Villaver, E., Stanghellini, L., \& Shaw, R.A. 2003, ApJ 597, 298

Waters, L.B.F.M., Molster, F.J., de Jong, T., et al. 1996, A\&A A 315, 361

Werner, M., Roellig, T. L., Low, F. J., et al. 2004, ApJS 154, 1 